\title{
'It has a lot more to contribute than just the teaching': Perceptions of participants in a peer-observation programme to develop clinical teaching
}

\author{
Authors: Louise Schofield and Michael Page
}

\section{Aims}

This study sought to evaluate the perceptions of participants in a faculty development project involving peer observation of clinical teaching. Learning in the workplace, and therefore clinical teaching, remain important for the development of doctors. It is recognised that clinical teaching is complex, and that doctors need support to develop the skills necessary to become effective educators. The literature suggests that doctors develop as clinical teachers through experiential learning based in the workplace, learning from peers and critical reflection, and this is best achieved through faculty development situated in the workplace where teaching occurs. Peer observation of teaching (POT) has been shown to be a means of delivering such developmental requirements in higher education, but there is limited evidence for its benefit for clinical teaching or its acceptability to participants who often cite time and work pressure as barriers to learning.

\section{Methods}

This study is set in a district general hospital where a programme to improve clinical teaching by consultants on ward rounds has been developing since 2015. This includes workshops on clinical teaching and the opportunity to have a consultant peer observe teaching on the participant's ward rounds. The observation is followed by a reflective discussion on what was observed. To date, consultants in acute medicine, paediatrics and obstetrics and gynaecology have participated in peer observation. As part of the programme evaluation, an interpretive phenomenological approach was used to understand participants' perceptions of the experience. Consultants who had taken part in an observation between 2 and 6 months previously were invited for a semi-structured interview. Data was recorded, transcribed verbatim and then analysed using inductive thematic analysis.

\section{Results}

Four interviews were completed. Consultants perceived that observation had improved their teaching skills and stimulated ongoing reflection on teaching. There was evidence of learning by observation as well as being observed. The role of the peer in guiding reflection and the development of relationships with other educators were highly valued. However, the role of the workplace community in supporting and sustaining learning was variable, and there was concern regarding the potential for evaluation rather than development. Time was felt to be a challenge which was surmountable.

\section{Conclusion}

POT is valued by participants as an acceptable means of developing clinical teaching and reflective practice. Building relationships and thus developing a community of educators seems possible. The role of the workplace community in supporting educators needs further exploration.

\section{Conflict of interest statement}

The lead author is director of medical education at the hospital site, and has developed the faculty development programme being evaluated. 